\title{
RESIDENCE EN PERSPECTIVE
}

\author{
JAMes Parkhouse, M.A., M.D., F.F.A.R.C.S., D.A."
}

\section{Deux catégories de personnel}

DANS UN SERVICE hospitalier et particulièrement dans une spécialté comme l'anesthésie où une grande partie du travail quotidien est d'ordre technique-on peut plaider en faveur de l'existence de deux types differents d'hommes dans le personnel du service: une élite intellectuelle qui assiste aux assemblées, aux congrès, qui rédige des articles et s'adonne à la recherche, puis un groupe de bons cliniciens honnêtes qui font l'ouvrage. Ce en quoi consiste "faire l'ouvrage" est une grande question; et ce qui peut raisonnablement, en médecine, être considéré comme une élite intellectuelle est une question difficile. Je me suis prononcé ailleurs sur les dangers d'une philosophie à deux cultures, avec la confrontation entre hôpital et université; et puisqu'il y a tant à dire concernant les qualifications des gens et leurs différences, c'est un bon point de départ.

Il nous faut des consultants qui soient des consultants. Leur fonction est de consulter. Toutefois, aujourd'hui, une grande partie du travail à faire auprès des malades n'est pas de cette sorte de travail; un fort pourcentage est du travail technique et demain ce pourcentage sera encore plus élevé. Qui doit faire ce travail? nous n'avons pas besoin d'un consultant senior pour installer toutes les injections endo-veineuses; ce serait un gaspillage injustifiable de ressources humaines que de lui faire faire ce travail. Pour faire ce travail, cependant, il faut quelqu'un et il en est ainsi pour un grand nombre d'autres tâches secondaires et cela requiert une surveillance appropriée. Parmi le personnel de l'hôpital et de l'université, il nous faut songer non pas à une ou à deux, mais à plusieurs catégories de personnel.

\section{Le résident et la somme d'ouvrage}

Si l'on considère la compétence des assistants, il existe une différence évidente entre les hôpitaux qui reçoivent des résidents et ceux qui n'en ont pas. En fait, de mille et une façon, les résidents participent au travail de l'hôpital et de ses médecins; on les rémunère pour leur travail et les résidents tirent de ce travail tout ce qu'il y a de précieux pour leur expérience. En Grand Bretagne, il a été question d'employer l'étudiant comme main d'œuvre à l'hôpital et pour cause, mais il est important d'insister sur le besoin de main d'œuvre et de réelle responsabilité clinique durant la formation. Le problème se pose lorsque l'utilisation des résidents se confine seulement aux exigences du service hospitalier.

\section{Qualité et quantité}

L'approbation d'hôpitaux pour la formation des résidents a, depuis longtemps, été basée sur ce que l'hopital peut offrir. Jusqu'ici, cela va; mais il ne faut jamais

\footnotetext{
-Doyen en médecine pour le post-doctoral Université de Sheffield, Angleterre. Autrefois professeur et directeur du département d'anesthésie, Université de Manitoba et chef anesthésiste, Winnipeg General Hospital.
} 
oublier que le premier facteur à retenir pour décider du programme d'un résident ou du nombre de résidents dans un programme, c'est: ce que l'hôpital peut offrir au résident et non ce que le résident peut offrir à l'hôpital. Toutefois, en pratique, il existe d'autres facteurs: les hôpitaux, les commissions hospitalières qui paient la note, réclament un droit de regard sur le travail des résidents. De plus, il y a une cote plus élevée dans le fait d’avoir des résidents; ce qui peut ramener à glisser sur certaines lacunes dans les conditions de travail offertes. Il y a même une cote encore plus élevée si l'on fait dire que les postes de résident sont tous remplis dans un département. Il est également nécessaire d'ouvrir un nombre suffisant de postes dans le pays pour satisfaire à la demande des étudiants en médecine locaux et à celle des étudiants étrangers.

La tendance actuelle s'oriente vers la reconnaissance des programmes de résidence universitaire plutôt que vers les différents hôpitaux et il est heureux que l'attention se soit portée vers le contenu académique et l'ensemble des activités de la résidence. Il est possible qu’en même temps plusieurs départements s'enquièrent plus sérieusement sur les intentions de l'étudiant étranger-pourquoi vient-il, où a-t-il l'intention d'aller, quels sont ses besoins spécifiques et qu'est-ce qui doit entrer dans l'ordre des choses. Pour ces deux raisons, il faut s'attendre à un déplacement de la motivation dans le choix de la résidence: elle ira de la quantité vers la qualité.

\section{Qui paie laddition?}

Quelque souriant que puisse paraitre l'avenir pour le valeureux corps académique, il faut faire attention de changer un mariage difficile pour un divorce catastrophique. Que les résidents soient payés par les hôpitaux pour faire l'ouvrage qu'il y a à faire dans l'hôpital, cela peut avoir des désavantages évidents, mais cela place la chose importante à sa place-sur l'expérience clinique-et cela vaut mieux que ne rien recevoir du tout. Si l'on met de l'avant que les programmes de résidence deviennent une affaire universitaire, il devient logique de se demander si les universités devront payer, au moins en partie, pour la formation. Mais les universités peuvent bien prétendre qu'elles offrent un précieux service en formant des résidents, ce qui est déja assez coûteux en efforts et en temps consacré de la part de la faculté, et pour lequel elle ne reçoit rien en retour, contrairement à l'hopital, service actuel et futur. En Grande Bretagne, le service national de santé est, en définitive, l'employeur des étudiants et des spécialistes en formation et le coût de la formation, même dans ses aspects académiques, devient un placement légitime à long terme du gouvernement. Là où c'est la pratique privée qui existe, il faut reconnaitre que la personne qui veut tirer un bénéfice de sa formation est le résident lui-même, et il ne peut que rarement faire face à un placement pour son avenir au point de se pourvoir à lui-même. A un tel dilemme je ne vois qưune réponse: le partage de la responsabilité.

\section{Seulement des chefs, pas d'Indiens}

$\mathrm{Si}$, d'une part, nous considérons la résidence comme une expérience à acquérir plutôt que comme un emploi dans un hôpital et que, d'autre part, nous essayons de trouver un mode de rémunération qui pourrait libérer le résident d'une partie 
de son travail à l'hôpital, il nous faudra faire face à la situation: qui va faire le travail clinique_et pas seulement la même somme d'ouvrage qu'auparavant mais encore davantage, il nous faudra anticiper un engagement beaucoup plus important dans l'enseignement. Placerons-nous tous les hôpitaux dans la situation où se trouvent les hôpitaux sans résidents où, de fait, tout geste médical, quelle que soit la dextérité ou l'expérience qu'il exige, est fait par des spécialistes? Allons-nous mettre sur pied une deuxième catégorie non en formation ou bien, après tout, tomber d'accord pour deux sortes de consultants?

\section{La tradition dindividu à individu}

Cette conception que le médecin assume la responsabilité personnelle de son malade est noble et respectée depuis toujours. Dans la pratique privée de la médecine il existe une croyance que si un malade vous choisit parmi tous les autres docteurs, comme médecin, alors vous seul devez en prendre soin. En temps ordinaire, on peut tenir compte de cette conception et l'appliquer presque à la lettre tout un demeurant assez efficace. Mais dans la pratique hospitalière d'aujourd'hui, il devient de plus en plus difficile de conserver une relation d'individu à individu entre le médecin et son malade. Un malade voit un chirurgien; le chirurgien considère l'individu comme son malade. Le malade est admis à l'hôpital, a besoin d'explorations radiologiques intensives, puis d'anesthésie et de chirurgie.

A qui appartient ce malade lorsqu'il est au département de radiologie ou lorsqu'il fait un collapsus circulatoire dous anesthésie? Puis, au cours des suites opératoires, s'il requiert des soins pour un infarctus du myocarde, à qui appartient le malade? Est-ce que le radiologiste, l'anesthésiste et le cardiologue sont tous, en quelque sorte, les serviteurs du chirurgien ou, en fait, le malade n'est-il pas traité par une équipe? Et si l'équipe peut se composer de plusiers spécialistes, ne peutelle pas inclure également des techniciens en cardiologie et peut-être aussi des assistants médicaux?

La conception d'individu à individu entraine beaucoup de confusion et d'ennuis. Savoir ce qui peut être confié à un assistant pose, sans doute, un grand nombre de problèmes mais ils sont encore plus évidents en pratique privée que dans un service national de santé; mais il ne fait aucun doute que toute la médecine modern doit se baser sur la conception du travail d'équipe. Il nous faut de réels consultants parce qu'il doit exister de la vraie consultation entre tous ceux qui sont responsables du confort du malade. Cette absence de consultants constitue la plus grande lacune de notre pratique médicale aujourd'hui.

Naturellement, c'est dans les endroits où le malade a besoin de soins plus intensifs que le travail d'équipe devient plus évident. L'évidente difficulté d'organiser une unité de soins intensifs sur une base d'honoraires à l'acte médical est une preuve de cette avance; mais cela devient la première faille dans un système qui contient ses déficiences face à la médecine de demain. En ce qui concerne l'anesthésie, il devient de plus en plus apparent que, dans l'équipe, il faut inclure le consultant, l'assistant, l'infirmière et le technicien. On ne doit pas poser la question: "qui va administrer cette anesthésie?" mais plutot: "avec la main d'œuvre à notre disposition, comment allons-nous traiter le plus efficacement possible tous les 
malades en détresse respiratoire et tous les malades actuellement inconscients ou menacés de le devenir?" Non pas individu à individu, mais individu à un grand nombre et plusieurs individus pour un seul.

\section{Les étudiants en médecine et la main d'œuvre}

Maintenant que nous avons fait part de la main d'œuvre, faisons entrer l'éudiant en médecine dans le tableau. Plusieurs spécialistés, la mienne y comprise, font face au problème du recruitement. Elles voient un manque de spécialistes; il existe une pénurie de spécialistes. Ils cherchent une réponse dans le recrutement, et y a-t-il meilleur endroit pour le faire qu'auprès de l'étudiant? L'impression qui prévaut est: si l'étudiant pouvait être mis en contact avec l'anesthésie, ou quelque spécialité que se soit, et cela autant que possible, si l'on réussit à lui montrer tout ce qu'elle peut offrir, il y a espoir qu'il puisse en faire son choix à sa graduation. En pratique l'argument a du sens. Cela donne des résultats. En anesthésie, il y a peut-être plus à dire que dans certaines autres spécialités à cause de l'immensité du problème; en Grande Bretagne, l'anesthésie est la plus vaste spécialité du service national de santé. Mais il y a d'autres facteurs.

Les étudiants en médecine sont sollicités de toutes parts, et les campagnes de recrutement, bien que menées habilement, ne sont pas justifiables. De plus, il y a une limite au débit total de nos écoles de médecine. Nous ne pouvons pas résoudre notre problème de main d'œuvre en doublant, en un soir, le nombre des médecins dans le pays; nous ne pouvons pas le résoudre ce problème en soutirant de Pierre pour donner à Paul. La vérité c'est qu'il peut y avoir plus de spécialistes en puissance que nous n'en avons besoin dans certains domaines, et il est certain qu'il devrait y avoir à l'école de médecine, un meilleure orientation en regard de la popularité des différentes spécialités; notre ultime espoir pour aplanir nos difficultés demeure dans l'utilisation la plus efficace des hommes et des femmes à notre disposition.

\section{L'assistant médical}

Depuis l'avènement du service national de santé en Grande Bretagne, on a fait plusieurs essais avec les titres d'assistant médical. Le premier a été lofficier médical senior de l'hôpital-un médecin diplomé travaillant dans une spécialité de l'hopital mais sans les qualifications post-doctorales d'un consultant. L'on a éprouvé des difficultés avec ce titre, et il est difficile d'en faire le bilan. Il serait peut être juste de dire que ce personnage était trop près du consultant pour être heureux de son poste; avec le temps, un grand nombre sont devenus consultants et le titre a perdu de la popularité. Récemment, sont apparus les assistants médicaux, mais ici le choix du nom n'était pas heureux et les postes ne sont pas populaires partout. Nous remarquons, au nombre des recommandations du rapport de la Commission royale sur l'éducation médicale, la proposition de deux catégories de médecins non en formation à l'hopital; cette fois-ci, ils s'appelleront consultants et spécialistes. Le but de cela est que le spécialiste pourra avoir une sécurité d'emploi et la latitude, après une formation post-doctorale, de s'acheminer vers le poste de Consultant s'il le désire. Reste à voir comment cela fonctionnera. L'important 
c'est qu'après vingt ans d'expériences, pas toujours heureuses, et après avoir tourné le problème sous toutes ses faces et l'avoir étudié sérieusement, la conclusion conduit au même point: il est nécessaire de créer une deuxième catégorie quelconque.

\section{L'assistant technique}

De nos jours, nous nous efforçons de penser non pas en termes de médecine mais en termes de sciences de la santé. Nous entendons parler d'auxiliaires médicaux, et des groupes de techniciens et d'infirmières techniciennes nous entourent. De plus en plus les infirmières sont des diplomées universitaires; les pharmaciens sont des diplomés. Nous ne savons plus ce qu'est réellement un docteur ou ce qu'est réellement une infirmière ou ce qui constitue réellement l'ouvrage qu'ils sont supposées faire.

Bien que quelques spécialités, telle que la radiologie, emploient des techniciens depuis plusieurs années, la pratique varie considérablement selon les pays: l'Amérique du Nord possède des techniciens en thérapie par inhalation alors que l'Angleterre n'en a pas; l'Angleterre a des sages femmes; les Etats Unis et les pays Scandinaves ont des infirmières anésthésistes.

Ce n'est pas la place pour une longue discussion d'un problème complexe. Il doit suffire d'insister sur la nécessité de ce qui s’appelle en termes populaires une réévaluation critique de quelques unes de nos pratiques et plus particulièrement de nos préjudices en ce qui concerne la cession d'une partie de l'ouvrage traditionnel d'un docteur à des assistants d'expérience qui n'ont pas nécessairement un diplôme de docteur (M.D.). Il est de beaucoup préférable d'agir ainsi que d'organiser des campagnes de recrutement pour employer des résidents comme main d'œuvre.

\section{Formation en vue de quoi?}

La réévaluation du rôle de docteur présuppose la réévaluation de sa formation. La formation en elle-même n'est pas un but. Ce n'est même pas une chose en ellemême. Personne d'entre nous n'a jamais fini sa formation. Notre but est de préparer des gens aussi bien et aussi efficacement que nous le pouvons pour qu'ils puissent assumer les responsabilités qu'on voudra leur confier. A tout instant, ces responsabilités doivent être à mesure de l'achèvement et de la dextérité de la personne qui les assume. Si l'on retourne à la formation en résidence et aux responsabilités des résidents il nous faut nous rappeler alors que nous envisageons une phase d'un long processus-celui de maintenir un service de santé convenable pour la communauté.

On en a assez dit déjà et on en a déjà assez écrit ailleurs pour qu'il ne reste aucun doute que nous vivons dans un monde en changement perpétuel. La médecine avance à une vitesse inégalée et dans des directions imprévues. Nous ne pouvons faire face à cette situation qu'en conservant une flexibilité, en nous-mêmes et dans la formation de la nouvelle génération. Conserver est un mot important, car ne l'oublions pas, les gens sont nés plus flexibles que nous ne pensons. Cela ne consiste pas tant à créer des esprits ouverts et de la largeur de vue qu’à éviter sa destruction par une endoctrination prédoctorale et une étroite formation de spé- 
JAMES PARKHOUSE: RÉSIDENCE EN PERSPECTIVE

cialiste. Le fait le plus important à se remémorer dans l'élaboration des programmes éducationnels est le fait qu'aucun de nous n'a reçu une formation qui est appropriée à ce que faisons aujourd'hui et que notre enseignement d'aujourd'hui à nos étudiants sera démodé demain. A moins, voilà, que nous leur montrions à penser.

\section{La conception académique}

Il existe un vieux débat sur la nature propre de l'éducation médicale qui concerne spécifiquement l'étudiant diplomé; ou bien nous formons des anesthésistes ou des chirurgiens dans un sens spécifique de travail comme nous formons des chauffeurs d'autobus ou bien nous faisons l'éducation des gens dans le véritable esprit général de l'université. D'où l'impression plutôt incertaine: est-ce que l'éducation du diplomé demeure dans le gyron des universités ou devrait-elle être une formation clinique de vocation?

Il est clair qu'il nous faut un compromis. Nous entendons dire maintenant que la fonction de l'école de médecine est de produire des docteurs d'un type unique mais aptes à plusieurs possibilités. Cela met l'accent sur la conception académique de former l'exprit plutôt que la conception de vocation de former des mains. Je crois que cela est juste, mais je crois aussi que dans un monde où de nouvelles spécialités vont naître selon toute vraisemblance alors que de vieilles spécialités vont disparaitre, nous avons besoin d'un grand nombre de nos diplomés les mieux préparés pour qu'ils deviennent eux aussi candidats à plusieurs possibilités. En réalité ce dont nous avons besoin ce sont des types d'esprit qui peuvent s'adonner à des problèmes nouveaux avec le support d'une équipe de spécialistes.

\section{Le vrai spécialiste}

Nous voici de nouveau à faire la distinction entre un spécialiste et un consultant. Le vrai consultant n'est pas un spécialiste; c'est un généraliste en habit de spécialiste, habit qu'il peut changer pour s'adapter à la situation. Le vrai spécialiste est celui qui ne remplit qu'une fonction de façon experte-les Smith dont la devise familiale "un ouvrage bien fait" donne une idée de son ambition.

Il y a beaucoup de courage dans les recommandations du Rapport de la commission britannique à l'effet que, durant les trois ans suivant sa graduation, le médecin devrait poursuivre sa formation professionnelle générale ce qui peut s'organiser de façon à préparer des gens pour les différentes carrières de spécialistes y compris la médecine générale. Au moins, cela prépare un homme à se qualifier pour un travail et à jouer son rôle dans la communauté; un certain nombre vont demeurer dans cette catégorie et vont tirer orgueil d'être de vrais spécialistes; d'autres vont vouloir encore plus d'expérience, en profondeur et en étendue, pour devenir des consultants. A tout prendre, il ne s'agit pas là d'êtres supérieurs ou inférieurs; il s'agit de différentes sortes de gens avec des personnalités différentes et un sens des proportions différent entre l'action, la conception et l'organisation de l'ouvrage des autres.

Etant donné que la formation du spécialiste devient plus longue et plus détaillée, les aspects généraux de l'éducation deviennent concentrés de plus en plus 
dans les premières années. Nous nous acheminons, et nous le savons, vers la production de gens qui sont de bons spécialistes mais qui par ailleurs savent peu comment profiter de la vie et apprécier ce que le monde a à offrir. Ce serait formidable si l'on parvenait de quelque façon à renverser l'ordre naturel des choses; on pense à la réputation des gens d'Hollywood qui vivent heureux et qui songent à se marier plus tard. $\mathrm{Si}$, seulement, nous pouvions installer les gens dans leur carrière et, alors, compléter leur éducation générale. Ce n'est pas un problème facile à résoudre; l'idée de l'éducation professionnelle suivie de l'éducation du consultant est un pas dans la bonne direction aussi longtemps que consultant veut dire ce qu'il devrait être et non pas seulement un spécialiste plus spécialisé.

\section{Nos besoins et les besoins de notre monde}

Dans la formation des résidents nous ne nous adressons pas seulement à nos diplomés mais aussi à de nombreux médecins venant d'outre mer pour compléter leur formation. On rencontre de grandes divergences de vue concernant ces diplomés étrangers. Leurs intentions sont différentes: les uns désirent se former et s'intaller au Canada; d'autres veulent se former et retourner dans leur pays; d'autres ne font pas part de leurs intentions et d'autres n'ont pas encore pris de décision. Autant de groupes différents et cela ne signifie pas que la qualité de formation devrait être basée sur une loi pour les riches et une autre pour les pauvres; c'est une extension de l'idée de la formation des résidents comme une phase dans un processus extensif. Comment pouvons-nous nous attendre qu'une résidence adaptée pour la pratique d'un spécialiste au Canada va convenir aux besoins des Indes ou de la Corée? En qualité de société évoluée, nous avons quelque chose à offrir dans le sens de formation post doctorale et, en conséquence, nous devons accepter une obligation envers des pays où les mêmes facilités de formation n'existent pas. Si nous acceptons cette obligation, cela souligne la question de plainte sur la qualité de la formation prédoctorale de certains étrangers qui viennent. Un pays en voie de développement peut bien avoir une école de médecine de moins bonne qualité; mais il peut avoir un grand besoin de médecins et il peut produire les meilleurs docteurs possibles dans les circonstances. Il peut également avoir un besoin urgent de spécialistes et, devant cette lacune admise rend plus importante la venue chez nous de ces candidats à la spécialisation. Il y aurait beaucoup à dire sur l'établissement de centres de formation post doctorale pour les médecins d'outre mer qui manifestent le désir de retourner dans leur pays. C'est la meilleure façon pour nous de satisfaire leurs besoins.

Les médecins étrangers qui ont l'intention ou qui espérent demeurer au Canada devraient être acceptés selon leurs capacités et recevoir une formation égale à celle que reçoivent les médecins canadiens. La première année de résidence sera souvent une période de probation et d'évaluation et cela doit être expliqué clairement. Remplir les cadres dans un programme de résidence en acceptant des médecins étrangers à peu près sans discrimination après avoir répondu aux demandes d'admission des nôtres nous reporte au conflit de la qualité de la quantité; cela laisse de côté la conclusion que cet article se proposait de discuter. 